\title{
Effect of cAMP on bovine oocyte maturation in vitro
}

\author{
Valeria Makutina ${ }^{1,2}$, Albina Isaeva ${ }^{1, *}$, Anna Krivonogova ${ }^{1}$, and Alexey Deykin ${ }^{1}$ \\ ${ }^{1}$ Ural Federal Agrarian Research Center of the Ural Branch of the Russian Academy of Sciences, \\ Yekaterinburg, Russia \\ ${ }^{2}$ Center of Family Medicine, Yekaterinburg, Russia
}

\begin{abstract}
Cyclic nucleotides cAMP and cGMP are among the main molecules that control the maturation of mammal oocytes. The in vitro simulated physiological oocyte maturation (SPOM) method was used to model cAMP accumulation in the oocyte. In the current study, we preincubated the oocytes of cows (not primed with FSH) with cAMP modulators: N 6,2'-O-dibutyryladenosine 3',5'-cyclomonophosphate (dbcAMP) and 3-isobutyl-1-methylxanthine (IBMX). The use of SPOM increased the yield of bovine blastocysts.
\end{abstract}

\section{Introduction}

During maturation, mammal oocytes are retained in the meiosis diplotene. At the same time, oocytes form specific large nuclei called germinal vesicles. Cyclic nucleotides cAMP and cGMP are the main molecules responsible for meiotic arrest [1].

The main source of cyclic nucleotides in the oocyte are the somatic granulosa and cumulus cells surrounding it [2]. Conversely, gonadotropins follicle-stimulating hormone (FSH) and luteinizing hormone (LH) control the somatic cells [2]. During the antral stage of folliculogenesis, FSH binds to receptors on somatic cells, modifies them, and also causes the expression of LH receptors and epidermal growth factor [3]. In addition, FSH affects the interaction of cells of the oocyte-cumulus complex with each other through gap junctions. It is via gap junctions that oocyte maturation is regulated by accumulation of cAMP, cGMP, metabolites and RNA in them [4]. High levels of cAMP inside the oocyte activate protein kinase $\mathrm{A}$, which prevents the activation of the maturation-promoting factor, keeping the oocyte in the M-phase [5].

It is known that during folliculogenesis oocytes consistently acquire the potential for development. Thus, oocytes from small antral follicles (2-4 mm) have a low ability to reach the blastocyst stage later in comparison with oocytes from large follicles [6,7]. Although some oocytes in small antral follicles are able to form a blastocyst, they require additional maturation [8].

Exogenous priming of FSH has a good effect on oocyte maturation. Oocytes obtained from cows that were injected with FSH before egg retrieval have a higher potential to form

\footnotetext{
* Corresponding author : isaeva.05@bk.ru
} 
good-quality embryos than oocytes obtained from cows without priming [9]. Nevertheless, when using ovaries obtained at slaughter, animals are usually not pre-exposed to FSH, and small antral follicles prevail in the ovaries. Therefore, the "pre-maturation in vitro" (preIVM) method, which simulates the natural physiological growth of cyclic nucleotides during follicle maturation, can increase the oocyte competence and, as a result, increase the number of good-quality embryos.

The standard method of oocytes maturation in vitro (in vitro maturation - IVM) consists in obtaining immature oocyte-cumulus complexes from antral follicles and their subsequent maturation in an environment with FSH and LH/HCG (Human chorionic gonadotropin) [10]. At the same time, FSH leads to a short-term cAMP increase in oocytes [11]. Gradually, the cAMP level decreases, and meiotic division resumes [11].

Maturation of oocytes by directly increasing the cAMP concentration (often in the form of an analog of N6,2'-O-dibutyryladenosine 3',5'- cyclomonophosphate (dbcAMP)) in oocyte-cumulus complexes was first proposed by Funahashi H. et al. [12]. Substances such as adenylate cyclase [13], forskolin [14], or 3-isobutyl-1-methylxanthine (IBMX) can be used as inducing agents [15]. One of these approaches is SPOM [15]. In this method, oocyte-cumulus complexes are exposed for several hours to an agent that causes a significant spike in cAMP concentration (pre-IVM), which blocks the resumption of oocyte meiosis. This is followed by the IVM phase itself with or without the addition of FSH [15].

\section{Materials and methods}

Collection of oocytes.

The cow ovaries were collected during the slaughter of cattle at a specialized slaughterhouse and transported to the laboratory with a temperature of 30-370C for 3-4 hours after receipt. In the laboratory, visualized follicles from 2 to $8 \mathrm{~mm}$ were aspirated using an $18 \mathrm{G}$ needle attached to a $5 \mathrm{ml}$ syringe. Aspiration of follicles directly on the site and transportation of oocyte-cumulus complexes in a buffer or medium is impractical, since it significantly reduces the competence of oocytes for further development due to the loss of follicular cGMP cAMP, deactivation of protein kinase A and irreversible resumption of meiosis [11]. The follicular fluid provides oocyte-cumulus complexes with nutrients. It was possible to obtain from 5 to 10 immature oocyte-cumulus complexes (OCC) from unstimulated ovaries (without FSH priming).

Oocyte in vitro maturation (IVM).

The first method of oocyte-cumulus complexes maturation. The IVM medium was a commercial Continuous Single Culture Complete (CSCC, Irvine Scientific, USA) medium developed for human in vitro fertilization procedures, with the addition of $50 \mathrm{mcg} / \mathrm{ml} \mathrm{HCG}$ (human chorionic gonadotropin, RF) and $0.5 \mathrm{mcg} / \mathrm{ml} \mathrm{FSH} \mathrm{(Gonal,} \mathrm{Serono).} \mathrm{The} \mathrm{OCC} \mathrm{was}$ washed twice with IVM medium and cultured in $300 \mu$ l of IVM medium coated with paraffin Oil for Tissue Culture (Sage, USA) in $35 \mathrm{~mm}$ Petri dishes at $38.5{ }^{\circ} \mathrm{C}$ in a humid atmosphere with $6.5 \% \mathrm{CO} 2$ for 24 hours.

The second method of oocytes maturation.

Oocyte-cumulus complexes were placed for 3 hours in a CSCC culture medium containing $1 \mathrm{mM} \mathrm{N6,2'-O-dibutyryladenosine} \mathrm{3',5'-cyclomonophosphate} \mathrm{(Sigma)} \mathrm{and} 0.5$ $\mathrm{mM}$ 3-isobutyl-1-methylxanthine (IBMX, Sigma). After this preincubation, the oocytecumulus complexes were placed in a CSCC medium with 0.1 IU FSH for 22 hours coated with paraffin Oil for Tissue Culture in $35 \mathrm{~mm}$ Petri dishes at $38.5{ }^{\circ} \mathrm{C}$ in a humid atmosphere with $6.5 \% \mathrm{CO} 2$.

Oocyte maturity was determined by the presence of the first polar body after removal of cumulus cells by denudation in a medium containing hyaluronic acid $(0.1 \%)$. The level of mature oocytes was about $70 \%$ of the total number of OCCs. 
Sperm preparation and in vitro fertilization (IVF).

After IVM, samples of ejaculated sperm from bulls were thawed and centrifuged in $3 \mathrm{ml}$ of $80 \%$ Percoll density gradient (Irvine Scientific, USA) for 10 minutes at $1500 \mathrm{rpm}$. The sperm precipitate after centrifugation was washed with a buffer medium with the addition of $5 \mathrm{E} / \mathrm{ml}$ of heparin for 5 minutes at $1000 \mathrm{~g}$.

The CSCC medium was used as the fertilization medium. The mature OCCs were washed twice with the fertilization medium and placed in Petri dishes under paraffin oil at $38.5^{\circ} \mathrm{C}$ and $6.5 \% \mathrm{CO} 2$ at saturated humidity. Spermatozoids were added into a medium with mature OCC at a concentration of $1.0-2.0$ x106 mobile spermatozoids $/ \mathrm{ml}$. The level of fertilized oocytes in this case was about $50 \%$ of the total number of OCCs. The number of fertilized cells was estimated after a day by the number of dividing embryos.

Cultivation to blastocysts in vitro

The CSCC medium was used as the culture medium. The embryos were cultured at 38.5 ${ }^{\circ} \mathrm{C}$ in $6.5 \% \mathrm{CO} 2$ with saturated humidity for 7 days.

\section{Results and discussion}

The results of the comparison of the two systems of maturation of bovine oocytes showed no difference in the formation of oocytes at the MII stage. In both systems, the maturation levels were $65-70 \%$. Nevertheless, the formation of blastocysts and their quality improved with the use of SPOM and reached 15-20\%, while after normal maturation the yield of blastocysts did not exceed $10 \%$. A crucial role belongs to the stage of cAMP accumulation in oocyte-cumulus complexes as well as to the extended cultivation time to fertilization, which is 27-28 hours with pre-IVM, exceeding the fertilization time with a standard IVM. SPOM simulates molecular signals that occur during oocyte maturation in vivo. In contrast, oocytes mature spontaneously in the standard IVM when they are removed from the follicular fluid, and a premature meiosis resumption occurs without a complex cascade of molecular signals, which reduces the competence of oocytes and threatens their further mitotic divisions.

Thus, the use of SPOM does not improve the maturation of oocytes to the MII stage, but increases the number of blastocysts formed.

The research is executed at the expense of a grant of the Russian scientific fund (project No. 19-76-10022).

\section{References}

1. W.K. Cho, S. Stern, J.D. Biggers, Journal of Experimental Zoology, 187, 383-386 (1974)

2. R.B. Gilchrist, J.G. Thompson, Theriogenology, 67, 6-15 (2007)

3. G.F. Erickson, C. Wang, A..J Hsueh, Nature, 279, 336-338 (1979)

4. D.L. Russell, R.B. Gilchrist, H.M. Brown, J.G. Thompson, Theriogenology, 86, 62-68 (2016)

5. T.A. Rose-Hellekant, B.D. Bavister, Mol Reprod Dev, 44 (2), 241-249 (1996)

6. M.A. Bagg, M.B. Nottle, D.T. Armstrong, C.G. Grupen, Reprod Fertil, 19, 797-803 (2007)

7. R. Marchal, C. Vigneron, C. Perreau, A. Bali-Papp, P. Mermillod, Theriogenology, 57, 1523-1532 (2002) 
8. P.J. Hendriksen, P.L. Vos, W.N. Steenweg, M.M. Bevers, S.J. Dieleman, Theriogenology, 53, 11-20 (2000)

9. S. Sugimura, N. Kobayashi, H. Okae, T. Yamanouchi, H. Matsuda, T. Kojima, A. Yajima, Y. Hashiyada, M. Kaneda, K. Sato, K. Imai, K. Tanemura, T. Arima, R.B. Gilchrist, Sci Rep, 7, 6815 (2017)

10. R.G. Edwards, Nature, 208, 349-351 (1965)

11. J.X. Li, G.K. Mao, G.L. Xia, PLoS ONE, 7 e37835 (2012)

12. H. Funahashi, T.C. Cantley, B.N. Day, Biol Reprod, 57, 49 - 53 (1997)

13. A.M. Luciano, P. Pocar, E. Milanesi, S. Modina, D. Rieger, A. Lauria, F. Gandolfi, Mol Reprod Dev, 54, 86 - 91 (1999)

14. Y.M. Shu, H.T. Zeng, Z. Ren, G.L. Zhuang, X.Y. Liang, H.W. Shen, S.Z. Yao, P.Q. Ke, N.N. Wang, Hum Reprod, 23, 504 - 513 (2008)

15. S. Sugimura, T. Yamanouchi, M.G. Palmerini, Y. Hashiyada, K. Imai, R.B. Gilchrist, J Reprod Dev, 64 (3), 233-241 (2018) 